\title{
eJRIEPS
}

Ejournal de la recherche sur l'intervention en éducation physique et sport

$29 \mid 2013$

Varia

\section{Émotions et transformation des connaissances en éducation physique : une étude de cas en kayak de mer}

Emotions and transformation of knowledge in physical education: a case study of sea kayaking

Nicolas Terré, Jacques Saury et Carole Sève

\section{(2) OpenEdition}

\section{Journals}

Édition électronique

URL : http://journals.openedition.org/ejrieps/2644

DOI : 10.4000/ejrieps.2644

ISSN : 2105-0821

Éditeur

ELLIADD

Référence électronique

Nicolas Terré, Jacques Saury et Carole Sève, «Émotions et transformation des connaissances en éducation physique : une étude de cas en kayak de mer », eJRIEPS [En ligne], 29 | 2013, mis en ligne le 01 avril 2013, consulté le 03 octobre 2019. URL : http://journals.openedition.org/ejrieps/2644 ; DOI : 10.4000/ejrieps.2644

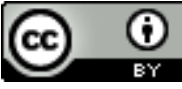

La revue eJRIEPS est mise à disposition selon les termes de la Creative Commons Attribution 4.0 International License. 
eJRIEPS 29 avril 2013

Émotions et transformation des connaissances en éducation physique : une étude de cas en kayak de mer

Nicolas Terré, Jacques Saury et Carole Sève

Laboratoire «Motricité, Interactions, Performance » EA 4334, Université Nantes Angers Le Mans, France

Résumé

Cette étude visait à caractériser des relations entre émotions et connaissances chez des élèves engagés dans la pratique du kayak de mer au cours de leçons d'Éducation Physique et Sportive (EPS). II s'agissait plus particulièrement d'étudier les processus de construction des connaissances en relation avec des modes d'engagement émotionnel repérés dans leur activité. En référence au programme de recherche du " cours d'action ", les phénomènes émotionnels ont été approchés du point de vue des acteurs, à partir d'enregistrements audio-visuels de leurs comportements et communications, et de verbalisations rétrospectives recueillies lors d'entretiens d'autoconfrontation. L'analyse révèle la manière dont les émotions ressenties participent à la construction de connaissances (a) en déclenchant une enquête, (b) en élargissant le champ des actions possibles, ou (c) en délimitant le champ des actions possibles. Ces résultats ouvrent de nouvelles perspectives d'analyse et de conception de dispositifs d'enseignement et d'apprentissage en EPS et à l'école en général.

Mots clés : apprentissage, émotions, connaissances, expérience, éducation physique

\section{Introduction}

Une des explications de la réussite ou de l'échec scolaire peut être recherchée dans les émotions vécues par les élèves lors de leurs expériences d'apprentissage. Celles-ci peuvent aussi bien fournir l'énergie essentielle aux apprentissages que perturber la progression des élèves (Ria \& Récopé, 2005). Les émotions deviennent pour de nombreux chercheurs et experts de l'enseignement scolaire un «pilier de l'apprentissage en classe " (OCDE, 2010a). Parmi ses recommandations aux politiques publiques en matière de conceptions souhaitables d'environnements d'apprentissage, le Centre pour la Recherche et l'Innovation dans l'enseignement (CERI) souligne l'importance de 


\section{eJRIEPS 29 avril 2013}

sensibiliser les enseignants aux influences des émotions positives et négatives sur l'apprentissage (Boekaerts, 2010). Selon les rédacteurs de ce rapport, les élèves qui éprouveraient des émotions positives seraient plus motivés à l'égard des activités scolaires et s'engageraient de manière plus active et constructive dans un apprentissage (Ryan \& Deci, 2000). A contrario, les élèves qui éprouveraient des émotions négatives pourraient se désinvestir de la tâche en cours, rediriger leur attention sur leurs émotions ou sur les caractéristiques défavorables de l'environnement d'apprentissage, et perdre confiance en eux (Covington, 1992 ; Weiner, 2007). Ces auteurs en déduisent que les émotions positives sont à encourager par les enseignants, et qu'en revanche les émotions négatives sont à réguler par les élèves, ces derniers pouvant être aidés par leurs enseignants quand ils ne parviennent pas à adopter des stratégies efficaces pour y parvenir.

Nous faisons l'hypothèse, dans notre étude, qu'une autre approche des relations entre émotions et apprentissage est possible. Les émotions et la construction de connaissances chez des élèves en Education Physique et Sportive (EPS) sont appréhendées de façon conjointe, sans dissocier a priori les phénomènes émotionnels et la dynamique de construction des connaissances ni préjuger de relations causales entre eux. Nous pensons qu'une telle démarche est de nature à révéler des phénomènes permettant de mieux comprendre les processus d'apprentissage et d'aider à la conception de dispositifs d'enseignement en EPS.

Le programme de recherche du « cours d'action » (Theureau, 2004, 2006) constitue le cadre à partir duquel nous avons analysé l'activité des élèves. Les connaissances y sont définies comme des expériences-types qui se construisent et s'actualisent dans un flux continu d'actions pratiques, de communications, d'interprétations, de focalisations, de perceptions et d'émotions significatives pour les acteurs (Theureau, 2006). Elles créent un ordre dans le flux de l'expérience (Varela, 1988) en établissant des liens entre des expériences remarquables. Elles s'expriment sur le mode de la typicalité (Rosch, 1978) et permettent ainsi de réduire un vaste ensemble d'expériences singulières en un ensemble plus limité de catégories. Cette conception expérientielle et dynamique des connaissances trouve un écho dans le contexte éducatif actuel. Comme dans les autres pays concernés par le Programme International de l'OCDE pour le Suivi des Acquis (PISA) (OCDE, 2010b), le système éducatif français a pour objectif de conduire un maximum d'élèves à réussir des tâches complexes. L'enjeu n'est pas pour les élèves d'accumuler des 


\section{eJRIEPS 29 avril 2013}

connaissances ; il s'agit surtout pour eux d'apprendre à les mobiliser dans des activités proches de celles rencontrées dans «la vie quotidienne » et dans lesquelles ils sont personnellement impliqués, intellectuellement et émotionnellement (Boekaerts, 2010 ; Masciotra \& Morel, 2011). Les dispositifs d'apprentissage scolaires peuvent alors s'inspirer de la recherche technologique en formation des adultes (Durand, 2008 ; Horcik \& Durand, 2011) et être analysés comme des « espaces d'actions encouragées » (Bril, 2002), c'està-dire des environnements d'apprentissage à la fois conformes aux modélisations d'une activité cible et dotés de propriétés particulières.

Le projet kayak de mer sur lequel était centrée notre étude répondait à ce double enjeu. Pendant une année, une classe de collégiens a vécu, dans le cadre de l'EPS, une pratique du kayak faisant référence à l'histoire du kayak arctique. Les élèves ont été initiés aux différentes techniques d'esquimautage et de propulsion que les chasseurs inuit avaient affinées au cours de siècles de pratique. La pratique du kayak de mer devait donner l'occasion aux élèves de s'ouvrir à la culture inuit, grâce à l'apprentissage de ces techniques traditionnelles. Au-delà des esquimautages, les élèves jouaient les rôles d'aide, de pareur, de juge, de reporter, de bloggeur, etc. Pour chacune de ces activités, les enseignants d'EPS et de français transformaient l'environnement des activités cibles (e.g., la réalisation de techniques traditionnelles d'esquimautages, la rédaction d'articles pour le blog de la classe, etc.) pour qu'ils constituent des « appels » et servent d'ancrage potentiel pour l'activité qui était souhaitée pour eux (Durand, 2008). Ces transformations de l'environnement pouvaient porter sur plusieurs aspects comme, par exemple, l'euphémisation des conséquences de l'action (e.g., par la présence d'un pareur lors des esquimautages), le ralentissement ou l'accélération du temps (e.g., par l'utilisation de bouées pour marquer un arrêt avant la phase finale de l'esquimautage), ou la sollicitation et l'amplification d'émotions (e.g., par la présentation collective et simultanée d'esquimautages) (Durand, 2008). Ce dernier aspect nous est apparu prégnant dans ce projet. Les émotions pouvaient, comme souvent en EPS, envahir les leçons (Durand, 2001). Certains élèves riaient ou se réjouissaient, d'autres pleuraient, s'inquiétaient. Ce projet constituait donc un terrain privilégié, pour (a) interroger la façon dont les connaissances se construisent au cours des expériences émotionnelles, et (b) mesurer l'intérêt d'«encourager» ou de «décourager» les émotions dans la conception de dispositifs d'aide à l'apprentissage. 


\section{eJRIEPS 29 avril 2013}

Plusieurs études conduites dans le programme du « cours d'action » (Theureau, 2006) ont montré l'intérêt d'envisager l'analyse de l'enchâssement-implication des émotions dans les actions et raisonnements dans des situations sportives ou d'enseignement en EPS. Ces études ont décrit la tonalité émotionnelle des actions, interprétations ou décisions des enseignants d'EPS débutants au cours de la conduite de leçons (Ria, Sève, Saury, Theureau, \& Durand, 2003 ; Ria, 2007), ou de pongistes experts au cours de matchs (Sève, 2001 ; Sève, Ria, Poizat, Saury, \& Durand, 2007). Dans ces différents cas, cette tonalité émotionnelle émergeait sur le fond d'expérience(s) émotionnelle(s) type(s) des acteurs, et participait étroitement à la construction de nouvelles connaissances relatives aux situations de classe, ou de match.

L'idée d'une co-définition des connaissances et des émotions est accréditée par les recherches actuelles en psychologie expérimentale et en neurosciences. Celles-ci considèrent que les cognitions et les prises de décision intègrent une composante émotionnelle dans la mesure où les émotions peuvent, (a) marquer les expériences passées (e.g., Damasio, 2010 ; Duncan \& Barrett, 2009 ; Phelps, 2006), (b) filtrer les perceptions actuelles (e.g., Berthoz, 2003 ; Bower, Monterot \& Gilligan, 1978 ; Fredrickson, 1998 ; Mogg, Millar \& Bradley, 2002), et (c) agir comme une mémoire implicite (e.g., Channouf, 2006 ; Damasio, 2010 ; LeDoux, 2003).

Notre étude visait, en s'inspirant de ces hypothèses développées dans d'autres programmes scientifiques que celui auquel nous nous référons, à caractériser les relations entre émotions et connaissances à un niveau phénoménologique. Elle s'est plus particulièrement attachée à caractériser les processus de mobilisation et de construction des connaissances en relation avec des modes d'engagement émotionnel repérés dans l'activité des élèves. Un tel éclairage est de nature à apporter une aide à la conception des dispositifs d'enseignement et d'apprentissage non seulement en EPS, mais à l'école en général.

\section{Cadre théorique}

Le programme de recherche du « cours d'action » pose l'hypothèse que toute activité est fondamentalement " cognitive, autonome, incarnée, située, individuelle-sociale, cultivée, vécue " (Theureau, 2004, 2006). De tels présupposés conduisent à mener une recherche à un niveau d'analyse où les personnes sont de véritables entités (Varela, Thompson \& 


\section{eJRIEPS 29 avril 2013}

Rosch, 1993) sans détacher a priori les phénomènes émotionnels des autres composantes de leur expérience (Saury, Adé, Gal-Petitfaux, Huet, Sève, \& Trohel, 2013).

1.1. L'étude des phénomènes émotionnels dans le cours d'action

Le programme du «cours d'action » (Theureau, 2006) propose un ensemble d'objets théoriques associés à des méthodes visant à les documenter et à les analyser dans le cadre d'enquêtes empiriques. Parmi les objets théoriques, le « cours d'expérience » rend compte du flux de significations (actions, communications, focalisations, interprétations et émotions « faisant signe ») émergeant de la dynamique du couplage entre un acteur et sa situation (Ibid.). Ce flux peut être étudié en relation avec les contraintes et effets extrinsèques de l'activité du sujet (i.e., la situation, la culture et l'état de l'acteur). Cette mise en relation délimite un second objet théorique : «le cours d'action ». La documentation de ces deux objets théoriques nécessite de définir un observatoire (Theureau, 2004). Celui-ci respecte des principes méthodologiques généraux (i.e., observations réalisées à l'issue d'une phase de familiarisation permettant d'obtenir la confiance des participants et de contractualiser la collaboration avec eux, enregistrements des comportements et communications en continu et in situ, recueil des verbalisations rétrospectives lors d'entretiens d'autoconfrontation), qui restent à spécifier dans chaque étude particulière selon les objets étudiés et les contraintes matérielles, institutionnelles, culturelles, propres à chaque situation (Sève \& Saury, 2010).

Un des principaux présupposés de ce programme est de considérer l'activité humaine comme autonome. L'idée fondamentale de la théorie de l'enaction qui est reprise ici est la notion de «clôture opérationnelle d'un système» (Varela, 1989). Chaque individu possède une capacité à faire émerger un monde qui est signifiant et pertinent sans qu'il soit prédéfini. Dans notre étude, cela signifie que les émotions vécues par les élèves, lorsqu'ils esquimautent en kayak de mer par exemple, dépendent de la façon dont ils définissent leur situation et des éléments du contexte qu'ils jugent significatifs. Les émotions possèdent une autonomie dont une analyse de la dynamique intrinsèque de l'activité peut rendre compte (Theureau, 2006). Un second présupposé consiste à envisager l'activité comme située. Les actions des individus sont indissociables et incompréhensibles en dehors des situations dans lesquelles elles s'accomplissent. Si les émotions portent l'empreinte des situations vécues par les élèves, elles participent en retour à l'évolution de ces situations. Du fait de la transformation permanente du couplage structurel acteur/environnement, les émotions des élèves sont donc à envisager comme 


\section{eJRIEPS 29 avril 2013}

des processus dynamiques. Enfin, un troisième présupposé considère l'activité comme une sémiose, c'est-à-dire comme une construction permanente de significations de la part de l'acteur. À chaque instant, l'activité s'accompagne d'une conscience préréflexive donnant lieu à expérience chez l'individu. Dans notre recherche, les sentiments - en tant qu'émotions émergeant dans la conscience préréflexive, donc donnant lieu à expérience sont considérés comme constitutifs de l'« effet de surface » de la dynamique du couplage de l'acteur à sa situation (Theureau, 2006). Ils constituent une part du cours d'expérience de l'acteur et peuvent être étudiés à partir de ce qu'il peut «montrer, raconter et commenter à tout instant » (lbid.).

Cependant, les émotions peuvent être ressenties sans toujours pouvoir être nommées. De plus, les labels verbaux ne peuvent refléter que ponctuellement une dynamique émotionnelle qui s'étale dans le temps (Dan Glauser, 2009). Plutôt que de documenter les émotions à partir des seules verbalisations des acteurs, il s'agit de les inférer à partir d'une analyse de la dynamique locale de leur cours d'expérience. Une telle démarche est de nature à conserver le caractère éminemment particulier, subjectif et dynamique des émotions.

1. 2. L'étude de la construction des connaissances dans le cours d'action La construction des connaissances peut être appréhendée sur la base de l'analyse de l'engendrement des signes et de leurs composantes au sein des « cours d'expérience » (Theureau, 2006).

L'expérience est discontinue. Un moment de conscience surgit, semble demeurer un instant, puis se dissipe pour être remplacé par le suivant (Varela et al., 1993). Les unités du cours d'expérience rendent compte de cette discontinuité. Chaque unité représente un signe dit hexadique dans la mesure où il est constitué de six composantes (Theureau, 2006). Chacune des composantes se réfère à une catégorie particulière de l'expérience de l'acteur tout en étant indissociable des autres. L'engagement de l'acteur (Engagement E) découlant des expériences passées ouvrent un champ de possibles en termes de préoccupations ou d'intérêts pratiques, qui délimitent à leur tour des attentes ou anticipations susceptibles de se réaliser dans la situation présente, compte tenu de cet engagement (Actualité potentielle A), et du fait de l'actualisation de certaines connaissances, ou éléments de généralité («actions-types », « situations-types », “ événements-types », etc.), issus des expériences passées (Référentiel S). Sur le fond de cette structure anticipative, s'actualisent des possibles en termes d'actions, de 


\section{eJRIEPS 29 avril 2013}

communications, de focalisations, d'émotions ou d'interprétations (Unité de cours d'expérience U), compte tenu de ce qui est jugé significatif par l'acteur et qui « fait choc » pour lui dans la situation (Représentamen $\mathrm{R}$ ), et pouvant donner lieu à la construction, la validation ou l'invalidation de connaissances ou «types " (Interprétant I). C'est cette notion d'interprétant qui permet de rendre compte du processus d'actualisationtransformation de connaissances usuelles, et de construction de nouvelles connaissances. Ce processus se fonde sur les hypothèses de "typification" ou «typicalisation» (Rosch, 1978 ; Theureau, 1992 ; Varela et al., 1993). Des types sont des éléments de généralité ou de familiarité qui émergent du couplage de l'individu avec son environnement. Ils servent implicitement de référence pour définir une catégorie de couplages action-situation.

L'enchaînement des signes permet de rendre compte de la transformation continue des significations en relation avec l'histoire du cours d'action passé et les événements rencontrés (Sève \& Saury, 2010). II est ainsi possible d'identifier la manière dont les éléments pris en compte $(R)$, l'unité élémentaire du cours d'action (U), et l'émergence / validation / invalidation de types (I) peuvent modifier les préoccupations de l'acteur (E), ses anticipations (A) et son référentiel (S) et définir les " ouverts " des signes suivants (Theureau, 2006).

L'ensemble de ces catégories analytiques, ainsi que le modèle générique de leur dynamique d'engendrement, ont orienté l'analyse des relations entre les phénomènes émotionnels et la construction de connaissances dans le cours d'expérience des élèves lors de leur pratique du kayak.

\section{Méthode}

2. 1. Situation étudiée et participants

Les situations étudiées s'inscrivaient dans un projet "Kayak arctique" articulant deux disciplines scolaires (EPS, français) et dont l'essentiel est consultable sur le blog de la classe : www.asqajaq.fr. Pour la partie EPS, deux objectifs étaient fixés pour ces élèves de troisième : progresser dans les esquimautages et déplacements et s'ouvrir à la culture inuit. Pendant une année, les élèves apprenaient à utiliser une pagaie groenlandaise. Comme dans les concours groenlandais, les esquimautages étaient envisagés comme une fin en soi, une occasion de défier les lois de l'équilibre, de reproduire de beaux gestes et de se lancer des défis. 


\section{eJRIEPS 29 avril 2013}

L'étude a été conduite en collaboration avec un enseignant d'EPS et treize élèves d'un même groupe de pratique. L'enseignant était volontaire pour accueillir le chercheur dans sa classe. Les objectifs de l'étude lui ont été présentés de manière globale sans que le thème ne soit précisé afin d'impacter au minimum sur ses pratiques d'enseignement et, plus largement, sur la pertinence écologique des situations étudiées. En concertation avec l'enseignant, deux élèves volontaires ont été retenus pour participer à des entretiens d'autoconfrontation. Ces derniers sont nommés par leurs pseudonymes inuit Lelouq et Paluk. Ils n'avaient jamais pratiqué le kayak de mer auparavant. Après cinq leçons, ils présentaient deux profils différents : Paluk était un garçon expressif, dont l'investissement en kayak de mer était fluctuant. II s'est trouvé assez vite en réussite au début de l'année scolaire, dans les esquimautages et dans les techniques de propulsion. Après la pause hivernale, son implication était devenue plus superficielle. Lelouq était une fille discrète, rigoureuse dans ses apprentissages en kayak de mer, et bonne élève en général. Son implication était constante depuis le début d'année. Elle avait déjà atteint un bon niveau de maîtrise en termes de navigation mais présentait une progression plus lente dans les esquimautages.

Une période de familiarisation avec le projet de la classe et l'activité des élèves sur l'eau a précédé l'étude. Des notes ethnographiques ont été prises au cours des neuf premières leçons. Cette «observation participante périphérique » (Adler \& Adler, 1987) (i.e., le chercheur a mis à profit l'opportunité qui lui était donnée de participer aux séances du fait de son statut d'enseignant dans le même établissement, sans pour autant intervenir directement dans la conduite des séances) a permis de suivre la progression de Lelouq et Paluk. Au cours de cette période, des entretiens préalables ont été menés avec eux. L'objectif était de leur permettre de se familiariser avec les entretiens d'autoconfrontation. Les élèves étaient aussi familiers des enregistrements vidéo, toutes les leçons étant filmées afin d'alimenter le blog de la classe. Ils pouvaient y écrire des commentaires. Nous avons consulté ce blog et nous sommes référés aux commentaires de Lelouq et Paluk lorsque ceux-ci nous permettaient de préciser leurs expériences vécues.

La leçon étudiée était la dixième d'un cycle comprenant au total quinze leçons. Elle s'est déroulée pendant deux heures sur une mer calme, puis légèrement plus agitée. Après avoir regroupé les élèves sur la plage, l'enseignant leur annonça qu'il souhaitait mettre avec eux l'accent sur l'esthétique des actions. II leur demanda de « réduire tous leurs gestes à leur plus simple expression » lorsqu'ils devaient embarquer, pagayer, s'allonger 


\section{eJRIEPS 29 avril 2013}

sur l'eau (Innaqatsineq), esquimauter avec la pagaie tenue à une main (Paatip kallua tuermillugu illuinnarmik) ou deux mains (Kinguffik paarlallugu), enchaîner rapidement des esquimautages (Nerfallarlugu assakaaneq), et débarquer. L'enseignant a regroupé ses élèves en « radeau » avant chaque nouvelle étape pour rappeler cette consigne, puis leur a apporté des aides ponctuelles (e.g., corrections verbales, manipulations) en intervenant auprès d'eux individuellement.

\section{2. Recueil de données}

Deux types de données ont été recueillis : (a) des observations et enregistrements audiovisuels en continu et in situ des comportements et communications, et (b) des verbalisations rétrospectives recueillies à partir d'entretiens d'autoconfrontation.

Les deux élèves ont été filmés durant l'intégralité de la leçon par une caméra embarquée située sur la proue du kayak. Une autre caméra a enregistré en plans larges les regroupements durant lesquels l'enseignant transmettait des consignes ou suscitait des échanges à propos des difficultés rencontrées ou des solutions trouvées. Les comportements (i.e., les mimiques et les réalisations motrices) et communications (i.e, les échanges avec l'enseignant et/ou d'autres élèves) ont été enregistrés et retranscrits verbatim. Ces enregistrements avaient deux fonctions. La première était de fournir aux élèves des traces de leur activité et de les y confronter lors des entretiens. La deuxième était de constituer un corpus de données sur le contexte et les actions des élèves afin d'aider à la documentation des « cours d'expérience ».

À l'issue de la séance, les deux élèves ont rédigé seuls un récit d'expérience. Il leur était demandé de faire apparaître ce qu'ils avaient fait, appris et ressenti au cours de la leçon. Les élèves s'étaient déjà exercés à cette pratique en cours de français pour publier des articles sur le blog de la classe. Les récits d'expérience ont servi de point d'ancrage pour les entretiens d'autoconfrontation qui se sont déroulés le lendemain de la leçon. Pour chaque élève, l'entretien a duré environ quarante-cinq minutes. II était demandé aux élèves de « revivre » et décrire leur activité lors de la séance telle qu'ils l'avaient vécue, sans chercher à l'analyser, la rationaliser ou la justifier a posteriori (Sève \& Saury, 2010). Chaque événement décrit dans le récit d'expérience a été interrogé avec pour matrice de questionnement les six composantes du signe hexadique. L'élève était systématiquement relancé sur ses actions pratiques, communications, interprétations, sensations, perceptions, focalisations et intentions. II a par exemple été demandé à Lelouq de se remettre dans la situation où elle s'était cognée la tête sur le kayak d'un de ses 


\section{eJRIEPS 29 avril 2013}

camarades et de décrire la scène : «Que cherches-tu à faire juste avant de te cogner la tête ? Que ressens-tu au moment du choc ? Te souviens-tu de ce que tu vois sous l'eau ? Où es-tu située par rapport à Philouk ? Te dis-tu quelque chose sous l'eau ? Qu'est-ce que tu essaies de faire ? » Dans cette première phase de l'entretien, des extraits verbatim des comportements et communications enregistrés in situ ont été repris par le chercheur afin de confronter l'élève aux traces de l'activité qu'il était en train de montrer, raconter ou commenter. Une fois le récit d'expérience «épuisé », chaque élève était interrogé sur les épisodes qu'il n'avait pas décrits spontanément dans son récit d'expérience en reprenant la chronologie de la leçon. Les questions s'appuyaient sur des communications et actions retranscrites dans le verbatim. II a par exemple été demandé à Paluk de commenter sa phrase «Merci Monsieur, vous m'avez sauvé deux fois la vie » après que l'enseignant l'ait aidé à se rétablir avec son kayak. Le chercheur relançait le discours des élèves selon les mêmes procédures.

2. 3. Reconstruction des cours d'expérience

La première étape du traitement des données a consisté à synchroniser, dans un tableau à deux volets (Tableau 1), la description des comportements de l'élève et de son environnement avec les verbalisations issues de l'entretien d'autoconfrontation. Les données d'observation et d'enregistrement ont été retranscrites dans la colonne de gauche : la localisation de l'élève sur le plan d'eau, sa distance ou proximité par rapport aux autres élèves, ses communications verbales et comportements. Celles-ci suivent la chronologie de la leçon. Les éléments du récit d'expérience et les verbalisations enregistrées au cours de l'entretien d'autoconfrontation ont été reportés dans la colonne de droite, en face des scènes qui sont montrées, racontées ou commentées. 


\begin{tabular}{|c|c|c|}
\hline \multicolumn{2}{|r|}{ Données d'observation et d'enregistrement } & Données d'entretien \\
\hline $16 h 43$ & $\begin{array}{l}\text { Une élève vient d'enchaîner deux esquimautages } \\
\text { rapidement. L'enseignant explique qu'elle a réussi } \\
\text { parce qu'elle s'est débrouillée pour faire } \\
\text { correspondre la fin du premier esquimautage avec } \\
\text { le début du second en revenant se pencher en } \\
\text { avant. } \\
\text { II propose à Paluk de venir à son tour essayer. } \\
\text { Professeur: "Paluk? Tu viens? " } \\
\text { Paluk regarde derrière. II hausse les épaules, met } \\
\text { sa cagoule et se rapproche du professeur. } \\
\text { Professeur: "Tu te mets face aux autres. " } \\
\text { II se place face au groupe. } \\
\text { II regarde l'enseignant et attend une vingtaine de } \\
\text { secondes avant d'esquimauter. } \\
\text { II se réajuste une dernière fois puis rentre dans } \\
\text { l'eau. II esquimaute une première fois et enchaîne } \\
\text { le second sans revenir complètement en position } \\
\text { de départ. Sous l'eau, il se bloque. II attend sans } \\
\text { déjuper en nageant avec le kayak vers } \\
\text { l'enseignant. L'enseignant lui présente la pointe de } \\
\text { son kayak. II prend appui dessus et se redresse } \\
\text { facilement en terminant avec le dos collé sur } \\
\text { l'arrière du pont. II met environ } 20 \text { secondes pour } \\
\text { se redresser. } \\
\text { Professeur : " Pourquoi il a manqué le deuxième? } \\
\text { Si ça a bloqué, c'est peut-être qu'il s'est trop } \\
\text { redressé. II n'était pas assez groupé pour } \\
\text { enchaîner le deuxième. " } \\
\text { Paluk : "Merci Monsieur, je vous dois deux fois la } \\
\text { vie. » }\end{array}$ & $\begin{array}{l}\text { Extrait du récit d'expérience : } \\
\text { « J'ai aussi essayé } \\
\text { d'enchaîner des } \\
\text { esquimautages mais je n'ai } \\
\text { pas réussi. » } \\
\text { Extrait de l'entretien } \\
\text { d'autoconfrontation: } \\
\text { «Moi aussi, j'ai fait le premier } \\
\text { esquimautage et j'ai raté le } \\
\text { deuxième. Même que la } \\
\text { deuxième fois, sous l'eau, je } \\
\text { ne savais pas si j'étais } \\
\text { complètement retombé. } \\
\text { J'étais un peu perdu la } \\
\text { deuxième fois sous l'eau. } \\
\text { Euh, sinon après... Sous } \\
\text { l'eau, la peur n'y est plus du } \\
\text { tout parce que je sais que le } \\
\text { professeur va arriver. Après } \\
\text { j'ai fait le petit chien et j'ai vu } \\
\text { le professeur arriver donc je } \\
\text { ne pense pas à la peur.... La } \\
\text { fois où il y avait les vagues il } \\
\text { m'avait sauvé une fois. Et là il } \\
\text { m'a sauvé une deuxième fois. } \\
\text { Je m'en rappelle tout de suite. } \\
\text { C'est toute cette séance où je } \\
\text { suis tombé trois fois qui est } \\
\text { claire. » }\end{array}$ \\
\hline
\end{tabular}

Tableau I. Extrait du protocole à deux volets réalisé pour l'étude du cours d'action de Paluk

La seconde étape a consisté à repérer et délimiter des "séquences émotionnelles 》 dans les cours d'expérience des élèves, sur la base du repérage de l'expression d'émotions dans les données d'enregistrement, d'observation et d'entretien. Par exemple, des expressions telles que "une peur de partir, de ne pas savoir se retourner ", "yes c'est génial! ", «ça m'a réconforté un peu du coup » ont servi de point de départ pour la documentation des signes des séquences émotionnelles du cours d'expérience de Paluk. 


\section{eJRIEPS 29 avril 2013}

Dix séquences émotionnelles ont été identifiées au total (Tableau 2). Elles ont été nommées de manière à restituer (a) le contenu qualitatif de l'émotion (en se référant aux termes employés par les élèves pour caractériser l'émotion ressentie), et (b) l'événement ayant engendré cette émotion. Ces séquences émotionnelles permettent de délimiter des moments au cours desquels des émotions ont été significatives pour les élèves (i.e., exprimées verbalement et/ou par des mimiques faciales), sans toutefois recouvrir l'ensemble des phénomènes émotionnels vécus au cours de la séance.

\begin{tabular}{|l|l|}
\hline \multicolumn{1}{|c|}{ Lelouq } & \multicolumn{1}{|c|}{ Paluk } \\
\hline L1 : Plaisir de réussir à pagayer les yeux & P1 : Crainte de devoir esquimauter \\
fermés & P2 : Longue hésitation avant \\
L2 : Plaisir de réussir Innaqatsineq & d'esquimauter \\
L3 : Peur suite au choc sous l'eau contre le & P3 : Joie de réussir son premier \\
kayak de Philouk & esquimautage \\
L4: Sentiment ambivalent entre & P4: Peur furtive liée au blocage sous l'eau \\
découragement et envie d'esquimauter & au cours d'un esquimautage \\
seule & P5: Peur de se faire renverser par les \\
L5 : Surprise par une vague venant sur le & vagues \\
côté & \\
\hline
\end{tabular}

Tableau II. Séquences émotionnelles identifiées pour Lelouq et Paluk

Pour chaque séquence émotionnelle, nous avons identifié trois étapes : l'ouverture, le développement et la clôture de la séquence. L'ouverture correspondait à l'apparition, dans une unité de cours d'expérience, d'un élément déclencheur d'une émotion significative (i.e., un signe dans lequel le représentamen engendrait, en relation avec la structure de préparation, le ressenti d'une émotion dans le signe suivant). Le développement correspondait à l'expression de l'émotion (i.e., les signes qui contenaient le ressenti, le renforcement ou l'atténuation de cette émotion). La clôture correspondait à la disparition de l'émotion ressentie (i.e., les signes au cours desquels les préoccupations et attentes déclenchées par l'émotion étaient satisfaites ou interrompues). Les composantes des signes hexadiques inclus dans ces séquences ont été documentées. Le Tableau 3 illustre 
eJRIEPS 29 avril 2013

les différentes étapes de la séquence émotionnelle P4 «Peur furtive liée au blocage sous l'eau au cours d'un esquimautage ». 
Signes hexadiques de Paluk

Signe 1 (ouverture de la séquence)

E : Enchaîner rapidement deux esquimautages

A : Position renversée

$\mathbf{S}$ : Sensations de renversement : le buste se retrouve vers la surface

$\mathbf{R}$ : Des perceptions inhabituelles

$\mathbf{U}$ : Ne sait pas s'il est renversé

I : Validation des types usuels

Signe 2 (développement de la séquence)

E : Se sortir de l'eau

A : La tête hors de l'eau

S : Expérience-type : le moment dans la $7^{\text {ème }}$ leçon où il avait réussi à se remonter en prenant appui sur le kayak du professeur

$\mathbf{R}$ : Le souvenir des sensations et actions réalisées au moment du sauvetage de la $7^{\text {ème }}$ leçon

$\mathbf{U}$ : Comprend qu'il s'est bloqué, ressent une peur très brève et pense à réaliser un sauvetage eskimo

I : Associe la situation actuelle et la situation vécue lors de la $7^{\text {ème }}$ leçon

Signe 3 (développement de la séquence)

E : Se sortir de l'eau sans déjuper

A : L'arrivée de l'enseignant

S : Actions-type pour réaliser un sauvetage eskimo : nager sans sortir du kayak, prendre appui sur la pointe d'un kayak

$\mathbf{R}$ : Le kayak du professeur

$\mathbf{U}:$ Fait le petit chien et voit le professeur arriver

I : Validation du type « sauvetage eskimo » avec le professeur

\section{Signe 4 (développement de la séquence)}

E : Se sortir de l'eau sans déjuper

A : Le redressement du kayak

$\mathbf{S}$ : Actions permettant de faciliter le redressement (dos collé au pont)

$\mathbf{R}$ : La pointe du kayak du professeur

U : Prend appui sur la proue du kayak du professeur et se redresse

I : Validation du type « sauvetage eskimo » avec le professeur

\section{Signe 5 (clôture de la séquence)}

E : Se rassurer

A : Un échange avec le professeur

$\mathbf{S}$ : La leçon où il avait réussi à se remonter en prenant appui sur le kayak du professeur

$\mathbf{R}$ : Bateau redressé sans déjuper. L'enseignant qui est venu le sauver.

U : S'adresse au professeur : «merci de m'avoir sauvé deux fois la vie »

I : Validation du type « sauvetage eskimo » avec le professeur

Tableau III. Documentation des signes hexadiques correspondant aux données présentées dans le Tableau 1 (séquence P4) et identification des éléments traduisant l'ouverture, le développement et la clôture de la séquence émotionnelle

\subsection{Analyse des relations émotions-connaissances}




\section{eJRIEPS 29 avril 2013}

Pour cette dernière étape, nous avons commencé par caractériser des « modes d'engagement émotionnel » sur la base de l'analyse des relations entre les différentes composantes des signes constituant chacune des séquences émotionnelles. Un mode d'engagement émotionnel correspond à une catégorie de préoccupations et d'attentes engendrées par le ressenti d'une émotion, et se prolongeant jusqu'à la clôture de la séquence émotionnelle. Par exemple, à partir des données présentées dans le Tableau 3 , nous avons caractérisé le mode d'engagement de Paluk comme une recherche de compréhension de ses perceptions inhabituelles et de sa peur.

Dans un deuxième temps, nous avons, pour chacune des séquences émotionnelles, analysé l'évolution des contenus du référentiel (i.e. les éléments de connaissances effectivement mobilisés) et de l'interprétant (i.e., les éléments de connaissances construits, renforcés ou invalidés).

Dans un dernier temps, nous avons caractérisé cette évolution des connaissances des élèves en relation avec les modes d'engagement émotionnel. II s'agissait de décrire la façon dont les modes d'engagement émotionnel pouvaient générer des modalités particulières de transformation des connaissances.

\section{Résultats}

Nous présentons les résultats en trois temps : la description (a) des modes d'engagement émotionnel identifiés, (b) des modes de transformation des connaissances, et (c) des processus émotionnels participant à la transformation des connaissances.

3. 1. Modes d'engagement émotionnel

L'analyse a permis de mettre en évidence trois modes d'engagement émotionnel : (a) donner du sens à l'émotion ressentie, (b) entretenir l'émotion ressentie, et (c) atténuer l'émotion ressentie. Plusieurs modes d'engagements émotionnels pouvaient être présents (successivement ou simultanément) dans une même séquence émotionnelle. Par exemple, lorsque Paluk ressentit de la peur en voyant de la houle (séquence P5), il donnait du sens à sa peur en se remémorant une expérience traumatisante, tout en cherchant à «s'en débarrasser». Le Tableau 4 présente les modes d'engagement émotionnel identifiés dans les dix séquences émotionnelles étudiées. 


\section{$\begin{array}{llllllllll}\text { L1 } & \text { L2 } & \text { L3 } & \text { L4 } & \text { L5 } & \text { P1 } & \text { P2 } & \text { P3 } & \text { P4 } & \text { P5 }\end{array}$}

\begin{tabular}{l}
\hline $\begin{array}{l}\text { Donner du sens à l'émotion } \\
\text { ressentie }\end{array}$ \\
\hline Entretenir l'émotion ressentie \\
\hline Atténuer l'émotion ressentie
\end{tabular}

Tableau IV. Identification des modes d'engagement émotionnel

\section{1. 1. Donner du sens à l'émotion ressentie}

Lorsque les élèves manifestaient ce mode d'engagement émotionnel, ils cherchaient à comprendre pourquoi ils avaient ressenti une émotion particulière, i.e., à interpréter la situation a posteriori en identifiant les éléments ayant généré l'émotion. Ce mode d'engagement a été identifié dans sept séquences émotionnelles. À titre d'illustration, il a été observé dans la séquence L5 "Surprise par une vague venant sur le côté ". Lelouq rentrait vers la plage, en pagayant avec fluidité comme l'avait demandé l'enseignant. Soudain, se sentant déséquilibrée dans son kayak, elle éprouva une légère peur. Tout en se rétablissant et en continuant à pagayer, elle a estimé qu'une vague avait failli la faire chavirer. Elle se demanda pourquoi et pensa, en regardant les vagues, que c'était sûrement parce que leur direction était de travers par rapport à la trajectoire de son kayak (« II y avait des vagues qui venaient comme ça [montre une direction avec sa main]. Et j'étais un peu comme ça [montre une direction perpendiculaire à la première avec son autre main]. »). Elle continua à pagayer en prêtant davantage attention à la direction des vagues. Au cours de cette séquence, l'émotion a joué un rôle d'alerte pour Lelouq. Celle-ci a pris conscience de l'existence d'une perturbation sans pour autant identifier instantanément l'événement qui l'avait causée. Suite à cette émotion, Lelouq a cherché à comprendre l'origine de cette perturbation.

\section{1. 2. Entretenir l'émotion ressentie}

Lorsque les élèves manifestaient ce mode d'engagement émotionnel, ils agissaient de manière à prolonger le plus longtemps possible une émotion agréable. Pour ce faire, ils modifiaient leurs attentes. Ce mode d'engagement a été identifié dans quatre séquences émotionnelles. À titre d'illustration, il a été observé dans la séquence L2 «Plaisir de 


\section{eJRIEPS 29 avril 2013}

réussir Innaqatsineq ». Lors d'un premier atelier, Lelouq et ses camarades devaient s'allonger sur le côté en amenant leur kayak sur la tranche (Innaqatsineq). Lelouq s'exerçait pour la première fois à ce demi-renversement en eau profonde et sans être assurée par un partenaire à terre. Elle travaillait avec son partenaire Philouk. Les deux élèves alternaient les rôles d'esquimauteur et de pareur sans sortir de leur kayak. L'enseignant leur avait demandé de donner une certaine « rondeur » au mouvement, c'est-à-dire de garder le kayak dans un même axe pour que les positions de départ et d'arrivée soient les mêmes. Les élèves devaient prendre un repère au moment de la descente et le retrouver une fois remontés ; le kayak ne devait pas pivoter. Philouk et Lelouq ont rapidement réussi. Après plusieurs essais, Philouk souhaitait arrêter l'exercice. Il demanda à Lelouq si elle aimait l'exercice. Elle lui répondit qu'elle « aimait bien ». Lelouq éprouvait du plaisir parce qu'elle appliquait correctement les consignes de l'enseignant («Innaqatsineq en restant à peu près droit. Ça j'ai réussi. II fallait revenir comme on était parti »). Puis, elle continua les renversements en se fixant de nouveaux buts («Quand je faisais Innaqatsineq j'essayais de ne pas faire trop de trucs qui ne servent à rien, de faire que ce qui est utile dans Innaqatsineq »). Ces critères renvoyaient à la notion d'épure (i.e., la réduction des gestes à leur plus simple expression). Ils avaient été abordés plus tôt dans la leçon mais non rappelés par l'enseignant pour cet atelier. La fréquence des essais de Lelouq augmentait et son sourire se lisait de plus en plus sur son visage jusqu'à ce que l'enseignant propose un nouvel atelier.

Tout au long de cette séquence, Lelouq a cherché à entretenir le plaisir qu'elle éprouvait. Elle a d'abord été satisfaite de réussir seule le basculement. Puis, elle a ressenti du plaisir quand elle a réussi à appliquer la consigne donnée par l'enseignant sur la « rondeur ». Suite à plusieurs réussites, Lelouq a cherché à prolonger cette émotion en modifiant à nouveau ses attentes en relation avec une autre consigne (i.e., l'épure) que l'enseignant avait donnée au début de la leçon.

\section{1. 3. Atténuer l'émotion ressentie}

Lorsque les élèves manifestaient ce mode d'engagement émotionnel, ils agissaient de manière à atténuer ou annuler une émotion ressentie comme désagréable. Ce mode d'engagement a été identifié dans quatre séquences émotionnelles. À titre d'illustration, il a été observé lors de la séquence P5 «Peur de se faire renverser par les vagues». Paluk rentrait vers la plage en fin de séance. L'enseignant venait de demander aux élèves de ne pas perdre de vue l'objectif de la leçon en éliminant tous les gestes parasites dans leurs 


\section{eJRIEPS 29 avril 2013}

coups de pagaie. Dès le début du trajet retour, Paluk perçut de la houle, et ressentit immédiatement de la peur. II se rappelait d'une autre séance au cours de laquelle les vagues l'avaient fait chavirer sur le trajet retour. Sa première préoccupation fut de ne pas tomber : il chercha d'abord à « regarder un peu les vagues pour essayer de les amortir ». Mais, plus il regardait les vagues, plus sa peur augmentait. Ses préoccupations se sont alors modifiées. Il ne cherchait plus seulement à ne pas tomber, mais surtout à « en finir avec cette peur ». Il essaya alors « d'avancer plus vite » pour terminer plus rapidement le trajet. Cependant sa peur persistait. II essaya ensuite de «parler à ses camarades pour ne plus penser à la peur ». En effet, Paluk avait l'habitude, pour surmonter son appréhension, de parler et de s'amuser avec ses camarades. Par exemple, en début de séance, il s'était amusé avec ses camarades pour ne pas penser aux ateliers d'esquimautage qu'il appréhendait. Cependant, dans le cas présent, le fait de parler à Engaroq n'atténua pas sa peur. II parla alors de sa peur à Engaroq et celui-ci lui proposa de pagayer dans la vague. II suivit ce conseil et, quelques coups de pagaies plus tard, il avait la sensation de mieux maîtriser son kayak et se sentait un peu plus en sécurité.

Tout au long de cette séquence, Paluk a tenté à plusieurs reprises d'atténuer sa peur. II a entrepris plusieurs actions. Ces dernières ne s'étant pas accompagnées d'une diminution de sa peur, il a suivi le conseil d'un camarade qui s'est montré, de son point de vue, efficace pour diminuer sa peur.

\section{2. Transformation des connaissances}

La caractérisation du référentiel et de l'interprétant des signes de chacune des dix séquences émotionnelles a mis en évidence l'importance des phénomènes de typification dans la transformation des connaissances. Trois modalités de construction des connaissances ont été observées: (a) le renforcement de types usuels, (b) l'affaiblissement de types usuels, (c) la construction de nouveaux types.

3. 2. 1. Le renforcement de types usuels

Dans cinq séquences émotionnelles, les élèves ont renforcé un ou plusieurs éléments de connaissance sur la base des émotions ressenties, soit parce que la mobilisation des types s'accompagnait d'un ressenti agréable, soit parce que la mobilisation des types permettait de réduire un ressenti désagréable. À titre d'illustration, ce renforcement de types usuels a été observé dans la séquence P4 «Peur furtive liée au blocage sous l'eau au cours d'un esquimautage ». Suite à son blocage, Paluk décida de réaliser un sauvetage eskimo (i.e., se redresser en prenant appui sur la pointe d'un autre kayak). En 


\section{eJRIEPS 29 avril 2013}

même temps que sa peur s'atténuait, différents éléments de connaissance s'actualisaient : le repérage sous l'eau, la nage d'attente, le redressement en prenant appui sur un kayak, la confiance accordée à l'enseignant. Paluk agissait dans la situation présente sur la base d'expériences émotionnelles passées qu'il reconnaissait comme analogues. II reproduisait des actions qui s'étaient antérieurement avérées efficaces dans ce type de situation. Du fait de leur efficacité dans la situation présente, les types mobilisés gagnaient en « force de conviction ».

\section{2. 2. L'affaiblissement de types usuels}

Dans deux séquences émotionnelles, les élèves ont diminué la « force de conviction » d'un ou plusieurs éléments de connaissance: leur mobilisation ne permettait pas de réduire un ressenti désagréable.

À titre d'illustration, cet affaiblissement des types mobilisés a été observé dans la séquence P5 «Peur de se faire renverser par les vagues». Paluk était tombé plusieurs fois à cause de la « houle arrière » lors d'une leçon précédente, et avait ressenti de la peur. Chargée d'émotion, la (re)connaissance de la « houle arrière », lorsqu'il rentrait vers la plage (lors de la leçon étudiée), l'a conduit à vivre la situation présente sur le fond de cette expérience passée. Celui-ci a alors reproduit des actions qui lui avaient permis d'atténuer sa peur lors de leçons précédentes. II essaya « d'avancer plus vite » puis de «parler à ses camarades pour ne plus penser à la peur ». Aucune de ces actions ne s'accompagna d'une diminution de sa peur : Paluk diminua la confiance qu'il accordait aux types mobilisés.

\section{2. 3. La construction de nouveaux types}

Dans cinq séquences émotionnelles, les élèves ont construit de nouveaux éléments de connaissance sur la base des émotions ressenties, soit parce que leurs actions s'étaient accompagnées d'un ressenti agréable, soit parce que leurs actions leur avaient permis de réduire un ressenti désagréable.

À titre d'illustration, cette construction de nouveaux types a été observée dans la séquence L2 «Plaisir de réussir Innaqatsineq». Alors qu'elle réussissait à s'allonger sur l'eau tout en conservant l'équilibre de son kayak, Lelouq se fixa comme but de continuer l'exercice en essayant de faire le moins de mouvements possible avec sa pagaie, en référence au critère d'épure annoncé par l'enseignant en début de leçon. Elle réussit. Son plaisir augmentait au fil des essais et un nouveau type se construisait. Celui-ci s'est d'ailleurs étendu à une nouvelle expérience, quarante-cinq minutes après cette séquence, 


\section{eJRIEPS 29 avril 2013}

lorsque Lelouq observait sa camarade Noemiq. Elle jugea spontanément de sa performance à l'aide de critères relatifs à la rondeur («Déjà elle arrivait à remonter et sinon son kayak restait à peu près dans le même axe »).

3. 3. «Processus émotionnels » de transformation des connaissances

La caractérisation des modes d'engagement émotionnel et l'analyse de la transformation des connaissances nous a permis de repérer trois processus par lesquels les émotions participent à la transformation des connaissances : (a) le déclenchement d'une enquête, (b) l'extension du champ des possibles, (c) la réduction du champ des possibles.

3. 3. 1. Le déclenchement d'une enquête

Dans deux modes d'engagement émotionnel («Donner du sens à l'émotion ressentie » et «Atténuer l'émotion ressentie »), la transformation des connaissances s'est produite sur la base d'un processus d'enquête visant, pour les élèves, à rechercher des éléments susceptibles (a) de donner du sens à l'émotion ressentie et/ou (b) d'atténuer l'émotion ressentie. Ressentant soudainement une émotion, ces derniers cherchaient à identifier des éléments significatifs de la situation pouvant en être à l'origine. Lorsque l'émotion ressentie perdurait et que les élèves tentaient de l'atténuer, ils reproduisaient des actions ayant déjà fait leur preuve et/ou exploraient de nouvelles solutions. Cette enquête s'accompagnait du renforcement, de l'affaiblissement ou de la construction de nouveaux éléments de connaissances.

A titre d'illustration, la persistance de la peur ressentie lors de la « houle arrière » a incité Paluk, après avoir mobilisé des types usuels s'étant avérés inefficaces, à s'engager dans une enquête et à tester de nouvelles actions. La peur qu'il ressentait a entretenu la dynamique de cette enquête jusqu'à ce Paluk suive le conseil d'un camarade permettant une meilleure maîtrise de son bateau et ainsi l'atténuation de sa peur. Du fait de son efficacité, ce conseil a permis la construction d'un nouvel élément de connaissance («Pagayer dans la vague permet de mieux contrôler son bateau »).

3. 2. 2. L'extension du champ des possibles

Dans deux modes d'engagement émotionnel («Donner du sens à l'émotion ressentie », «Entretenir l'émotion ressentie »), la transformation des connaissances s'est produite selon un processus d'extension du champ des possibles. Lorsque leurs attentes étaient satisfaites, les élèves se donnaient de nouveaux défis pour entretenir l'émotion ressentie. Ils répétaient les actions ayant généré l'émotion ressentie tout en se fixant de nouveaux 


\section{eJRIEPS 29 avril 2013}

critères de satisfaction. La réalisation de ces défis s'accompagnait de la construction de nouveaux éléments de connaissance.

A titre d'illustration, après que Lelouq ait éprouvé de la satisfaction à réussir Innaqatsineq seule (séquence L2), elle s'est successivement fixée deux nouveaux défis: le premier étant de réussir à se basculer et remonter sans faire pivoter le kayak (i.e., donner de la rondeur au mouvement) ; le second étant de réussir à se maintenir à la surface en faisant un minimum de mouvements (i.e., épurer au mouvement). Du fait de sa réussite, Lelouq a construit au cours de cette séquence deux nouveaux éléments de connaissance.

3. 2. 3. La réduction du champ des possibles

Dans deux modes d'engagement émotionnel («Donner du sens à l'émotion ressentie », «Atténuer l'émotion ressentie »), la transformation des connaissances s'est produite selon un processus de réduction du champ des possibles. L'émotion était ressentie sur la base de la remémoration d'une expérience émotionnelle passée. Des éléments de connaissances s'imposaient et conduisaient (a) à la «typicalisation émotionnelle » de la situation présente et (b) à la mobilisation d'actions-types qui avaient été jugées efficaces dans ce type de situation. L'expérience émotionnelle type, avec les éléments de connaissance qui s'y rattachent, pouvait ainsi être renforcée.

À titre d'illustration, lorsque Paluk s'est retrouvé bloqué sous l'eau entre deux esquimautages (séquence $\mathrm{P} 4$ ), les différents éléments de connaissance mobilisés (repérage sous l'eau, nage d'attente, redressement par prise d'appui sur un kayak, etc.) étaient liés entre eux par l'expérience émotionnelle de peur dans laquelle ils avaient été vécus. Revivre ce type de situation a conduit Paluk à mobiliser ces éléments comme un tout. La stabilité des relations entre actions-types et perceptions-types a limité les éléments de connaissance susceptibles d'être renforcés.

\section{Discussion}

Nous discutons les résultats de cette étude à deux niveaux, respectivement liés à ses enjeux scientifiques et professionnels. Dans la première partie, nous proposons des éléments d'interprétation de la façon dont les émotions participent (au niveau où elles sont significatives pour les élèves) à la dynamique de l'activité et de l'apprentissage des élèves. Dans la deuxième partie, nous envisageons des perspectives pédagogiques pouvant guider la conception de dispositifs d'apprentissage inspirés par ces interprétations. 


\section{eJRIEPS 29 avril 2013}

4. 1. Participation des émotions à la dynamique de l'activité et de l'apprentissage des élèves

Cette étude accrédite l'idée que les composantes émotionnelles de l'expérience des élèves en kayak de mer sont consubstantielles de leur activité et de leurs apprentissages car, (a) elles constituent des éléments de leur «structure de préparation » à chaque instant, jouant un rôle essentiel dans la façon dont les élèves font émerger «leur situation » en relation avec leurs expériences passées ; et (b) elles participent étroitement aux processus d'apprentissage chez les élèves, en « marquant » particulièrement certains processus de transformation des connaissances.

\section{1. 1. Les émotions participent à définir « la situation de l'élève »}

Cette étude montre que les émotions ressenties participent à la définition de la situation par l'élève en modifiant sa structure de préparation, c'est-à-dire son engagement, son actualité potentielle et son référentiel.

En effet, nous avons caractérisé, dans l'expérience des élèves, trois modes d'engagement émotionnel, visant respectivement à donner du sens à l'émotion ressentie, à entretenir l'émotion ressentie ou à l'atténuer. Ces modes d'engagement émotionnel mettent en évidence que les émotions sont l'objet de préoccupations particulières des élèves, visant à en interpréter l'origine ou à les contrôler (en cherchant à les faire durer ou à les atténuer), et délimitent dans le même temps un champ d'attentes particulières, en termes d'expériences émotionnelles possibles, ou plus ou moins précisément attendues. Par ailleurs, l'analyse montre que les élèves mobilisent à chaque instant des connaissances dont certaines sont liées à des expériences émotionnelles antérieures (e.g., la reconnaissance d'une situation-type de « retour vers la plage avec de la houle arrière », étroitement associée chez Paluk à un sentiment-type de peur de chavirer). Les composantes émotionnelles de l'expérience des élèves en kayak de mer peuvent, à ce titre, être considérées comme «encapsulées » dans les éléments de la «structure de préparation » des élèves à chaque instant du déroulement de leur activité, ce qui met en évidence leur caractère « anticipatoire », ou «pro-actif ». L'analyse montre enfin que les élèves, en agissant «avec » et « sur » leurs émotions, ont également des attentes actives vis-à-vis de leurs émotions. Ils ne se limitent pas à s'adapter de façon « réactive » à des événements perçus comme menaçants (sur un mode relativement passif), ce mode réactif apparaissant toutefois dans certaines séquences émotionnelles lorsqu'un élément de la situation (e.g., la sensation d'un déséquilibre) fait «choc » de façon particulièrement 


\section{eJRIEPS 29 avril 2013}

inattendue. L'émotion émerge de ce qui fait signe pour l'acteur (i.e., l'objet de l'émotion), dans sa situation, compte tenu de ses expériences émotionnelles passées, et évolue en revenant sans cesse sur son objet, qui lui-même évolue avec la situation de l'acteur (e.g., le plaisir ressenti par Lelouq en réalisant Innaqatsineq évolue avec ce qui est le signe, pour elle, d'une bonne réalisation).

Cette analyse élargit l'interprétation de la place des émotions dans l'activité des élèves, par rapport aux conceptions qui les envisagent, soit comme une alerte provenant d'une distorsion entre la situation et les attentes de l'acteur (Frijda, 1986 ; Livet, 2002), soit comme une réorganisation de l'activité en réponse à l'évaluation d'un stimulus interne ou externe (Grandjean \& Scherer, 2009). Elle concourt à accréditer l'idée selon laquelle les émotions constituent un « ressort pour l'action» (Ria \& Récopé, 2005), voire que les émotions peuvent elles-mêmes être considérées comme des « actions pour modifier notre rapport au monde » (Sartre, [1938] 1995). En effet, les émotions vécues par les élèves en kayak de mer visaient autant à modeler le présent et anticiper le futur qu'à évaluer le passé. Nos résultats convergent, en ce point, avec des hypothèses développées en neurophysiologie selon lesquelles les émotions sont une «préparation à agir » en aidant le cerveau à catégoriser le monde et à simplifier la neurocomputation en fonction des expériences passées (Berthoz, 2003). Selon Damasio (2010), il est heureux que nous puissions nous appuyer sur des épisodes clés de notre vie, en particulier sur des épisodes émotionnels, pour anticiper l'avenir sans avoir à «traiter » in extenso le contexte. Des affects, enfouis ou incorporés dans la connaissance que nous avons du monde, (Barrett, 2008) nous permettent de faire émerger ce qu'Edelman (1987) nomme le « présent rappelé ».

\section{1. 2. Les émotions participent à la transformation des connaissances}

Les résultats de cette étude mettent également en évidence que les émotions participent étroitement aux processus d'apprentissage et de construction de connaissances chez les élèves. Elles jouent en effet un rôle central dans les différentes modalités de renforcement ou d'affaiblissement de la force de conviction de connaissances héritées des expériences passées, ou de construction de connaissances nouvelles, en d'autres termes, dans les processus de typification. Les émotions «marquent » également de façon originale les phénomènes accompagnant cette transformation des connaissances, et que nous avons décrits en termes de « déclenchement d'une enquête », d' « extension du champ d'actions possibles », ou de « réduction du champ d'actions possibles ». À ce titre, les émotions 


\section{eJRIEPS 29 avril 2013}

constituent en elles-mêmes des éléments de connaissance, en même temps qu'elles orientent le processus d'apprentissage lui-même.

Nos résultats convergent avec l'idée esquissée par Sartre ([1938] 1995) selon laquelle les émotions englobent, dans une même synthèse, le sujet ému et l'objet émouvant. Dans l'expérience des élèves en kayak de mer, ces synthèses ont pris la forme d' "émotiontype » (e.g., le sentiment de peur associé à la (re)connaissance de la houle chez Paluk), d' « expérience émotionnelle type » (e.g., les actions de Paluk lors du sauvetage eskimo tenus par une expérience de peur) ou de « types affaiblis ou renforcés par des jugements affectifs » (e.g., le renforcement de l'importance de la rondeur dans la réalisation d'Innaqatsineq chez Lelouq associé à un sentiment de plaisir). Ainsi, le rôle des émotions dans les apprentissages ne peut être réduit à la facilitation ou à la perturbation de la transformation des connaissances. Nos résultats accréditent l'hypothèse d'une codéfinition des émotions et des connaissances. En plus des jugements rationnels, des jugements affectifs influent sur la transformation des connaissances. Pour certains neurophysiologistes, ces jugements affectifs apporteraient un «soi " à la connaissance (Damasio, 2010), une subjectivité nécessaire à la connaissance (Duncan \& Barrett, 2009). Cela corrobore l'idée selon laquelle la « force de conviction » des connaissances n'est pas seulement liée au nombre de situations dans lesquelles elles se sont vérifiées, mais aussi à la tonalité émotionnelle des situations dans lesquelles elles se sont constituées (Sève, 2001 ; Sève, Saury, Theureau, \& Durand, 2002a). Par exemple, lorsque les acteurs jugent la situation comme non typique, ils tendent à valider rapidement les actions satisfaisant aux exigences de l'activité en cours (Sève, Saury, Theureau, \& Durand 2002b). Comme dans l'exemple de Lelouq qui est surprise par une vague, l'élément essentiel, pour valider une nouvelle connaissance, est l'efficacité pragmatique (e.g., éviter de naviguer avec les vagues de travers pour ne plus se faire surprendre). C'est aussi ce que tendent à montrer des études portant sur la mémoire. Dans une recherche menée à la suite des attaques terroristes du 11 septembre 2001 auprès d'étudiants américains (Talarico, \& Rubin, 2003), il est apparu que ces derniers étaient en mesure, plusieurs semaines plus tard, d'évoquer des souvenirs à propos de cette journée de manière détaillée et avec une grande confiance. Cependant, ces souvenirs n'étaient pas forcément exacts. La composante émotionnelle des connaissances agirait, non pas sur l'exactitude de la mémoire, mais plutôt sur le sentiment de savoir (Phelps, 2006). Une plus grande confiance serait donc accordée aux connaissances lorsqu'elles sont construites ou renforcées au cours 


\section{eJRIEPS 29 avril 2013}

d'expériences émotionnelles intenses (e.g., la confiance en la manœuvre du sauvetage eskimo chez Paluk).

\section{2. Perspectives pédagogiques}

Les relations que nous avons mises en évidence entre les émotions et les connaissances peuvent, en forçant certains traits, favoriser la compréhension de phénomènes observés dans d'autres contextes de pratique et donner lieu à des perspectives en termes d'enseignement. Nous avançons trois perspectives pédagogiques: (a) l'exploitation des émotions comme amplificateurs d'expérience, (b) la diversité des émotions à susciter, et (c) l'ouverture d'espaces de débriefing.

\section{2. 1. L'exploitation des émotions comme amplificateurs d'expérience}

La participation des émotions aux jugements de typification montre l'intérêt, pour les enseignants d'EPS, de solliciter et d'amplifier les émotions des élèves dans des « espaces d'actions encouragées » (Durand, 2008).

Un premier levier pédagogique peut consister, selon nous, à laisser une marge d'autonomie aux élèves afin qu'ils puissent générer des challenges stimulants pour eux et s'engager dans des défis significatifs. C'est par exemple ce que Lelouq a cherché à faire lorsqu'elle s'est fixée de nouveaux critères de satisfaction pour entretenir son plaisir dans la réalisation d'Innaqatsineq. Cette propension des élèves à faire évoluer les tâches en fonction de leurs préoccupations a déjà pu être observée chez des élèves en badminton (Saury \& Rossard, 2009). Elle nous semble pouvoir être anticipée et encouragée dans des espaces de défis permettant aux élèves de « régler » les tâches afin qu’ils puissent vivre et « capitaliser » des expériences émotionnelles.

Un deuxième levier pédagogique consiste à encourager des émotions pour amplifier les expériences des élèves, et par conséquent aider à la typification. Le « jeu » d'un élève dans un espace d'actions encouragées peut le conduire à éprouver des émotions et donner une subjectivité (un «je ») aux actions réalisées. Un tel dispositif a été mis en œuvre en kayak de mer lorsque les élèves apprenaient, lors de la deuxième leçon, à esquimauter avec des bidons au lieu d'une pagaie. Les bidons apportaient un supplément de flottabilité et encourageaient les élèves à balayer la surface de l'eau en s'éloignant au maximum de l'axe du kayak pour se redresser. La plupart des élèves s'amusait et réussissait à esquimauter. Ce jeu constituait ainsi un « espace d'actions encouragées », non pas seulement dans le sens d'une «incitation » à réaliser certaines actions, mais 


\section{eJRIEPS 29 avril 2013}

aussi probablement d'une « amplification » de la confiance accordée aux actions réalisées du fait des émotions ressenties.

\section{2. 2. La diversité des émotions à susciter}

La mise en évidence de différents « processus émotionnels » influant sur la transformation des connaissances souligne l'intérêt, pour les enseignants d'EPS, d'élargir les émotions à encourager au-delà du seul plaisir.

Nos résultats mettent en évidence que les émotions vécues par les élèves, même lorsqu'elles sont décrites comme «négatives» (e.g., la surprise de Lelouq qui a été déséquilibrée par une vague, le blocage de Paluk sous l'eau), ont une fonction adaptative. Ces résultats convergent avec ceux d'études menées dans le monde de l'entreprise et du travail (e.g., Tran, 2009 ; Marc, Grosjean et Marsella, 2011) où le salarié a parfois besoin de ressentir des tensions et d'identifier leurs causes pour s'adapter et gagner en efficacité. À un continuum d'émotions qui iraient du déplaisir au plaisir, et dont les propriétés seraient respectivement l'inhibition et la facilitation des apprentissages, nos résultats tendent au contraire à accréditer l'idée que toute émotion ressentie (de tonalité positive ou négative) peut a priori engendrer une transformation des connaissances dans le sens d'une adaptation «majorante » (même si, évidemment, cela n'est pas systématique). II n'y a donc pas à limiter les émotions favorables aux apprentissages scolaires au seul plaisir. S’il est important que les enseignants soient sensibles au plaisir ressenti par leurs élèves (BuiXuan \& Haye, 2007 ; Boekaerts, 2010), « cultivent » le plaisir (Gagnaire \& Lavie, 2005, 2010), ou s'engagent dans une véritable « didactique du plaisir » (Delignières, 2003), cela ne doit pas pour autant les conduire à « aseptiser » leurs dispositifs et empêcher que des émotions « négatives » ne soient vécues par les élèves.

En outre, il nous semble essentiel, pour que les élèves apprennent à agir en situation d'urgence, d'ajuster la « distance émotionnelle » entre les dispositifs d'apprentissage et la «pratique cible » (Durand, 2008 ; Horcik \& Durand, 2011). Cette « bonne distance » nous semble avoir été trouvée, par exemple, lorsque Paluk eut recours pour la première fois au sauvetage eskimo dans des conditions difficiles de navigation. Son immersion dans la «pratique cible » était à la fois « authentique », parce que la chute était imprévisible, et « guidée », parce que l'enseignant se trouvait à ses côtés pour le conseiller. Différents éléments de connaissance se sont agrégés au cours de cette expérience émotionnelle. Ils étaient tenus entre eux comme une « constellation de types » (Dougherty, 1985 ; 


\section{eJRIEPS 29 avril 2013}

Theureau, 2004) qui s'est actualisée « en bloc » lorsque Paluk (re)connut le même type de situation émotionnelle dans la leçon étudiée.

\section{2. 3. L'ouverture d'espaces de débriefing}

Les émotions pouvant devenir l'objet des préoccupations des élèves, il nous semble prometteur d'ouvrir, dans les dispositifs d'apprentissage, des «espaces de débriefing " dans lesquels les élèves peuvent exprimer et partager leurs émotions.

En effet, nos résultats montrent que les élèves, en cherchant à donner du sens ou à atténuer l'émotion ressentie, transforment leurs connaissances. L'enseignant peut donc encourager ces modes d'engagement en incitant les élèves à raconter leurs expériences émotionnelles. Au-delà d'une posture empathique permettant à l'enseignant d'être réceptif aux expériences émotionnelles des élèves, il est possible de s'inspirer de trois procédures exploitées dans cette étude pour documenter l'expérience des élèves: le récit d'expérience, le blog de la classe, et les échanges spontanés dans des dispositifs collectifs d'apprentissage.

Un premier intérêt, pour les élèves, de pouvoir exprimer leurs émotions, est d'expliciter la signification qu'ils accordent aux événements émotionnels. Le blog de la classe et les récits d'expérience sont deux procédures qui nous semblent avoir joué ce rôle dans cette étude. En exprimant ce qu'ils avaient vécu, les élèves mettaient des mots sur ce qu'ils avaient senti et appris. L'ouverture d'espaces de débriefing présente également l'intérêt, pour les élèves, de pouvoir solliciter l'aide d'un pair lorsqu'ils ne parviennent pas à contrôler une émotion ressentie comme désagréable. La mise en place de dispositifs collectifs d'apprentissage nous semble avoir tenu ce rôle dans la leçon étudiée. Ces espaces de débriefing au cours duquel les élèves sont incités à exprimer et partager leurs émotions peuvent en outre être rapprochés de la procédure dite des "coping modèles " (Lafont, Cicéro, Martin, Vedel, \& Viala, 2005), dans laquelle un élève tuteur exprime ses émotions au cours de l'apprentissage, et tient compte des émotions de l'élève apprenant. L'efficacité de cette procédure (en termes d'apprentissage et de développement du sentiment d'efficacité personnelle des élèves) comparativement aux formes plus classiques de tutelle, est convergente à nos yeux avec l'intérêt de tels débriefings. Mettre en mots ses émotions et accéder aux émotions des autres, liées aux situations d'apprentissage, aide à apprendre. La pertinence des espaces de débriefing semble liée au fait que les élèves peuvent y raconter leurs expériences sans avoir à les emprunter ou les copier sur autrui, ni se sentir obligés de rappeler les consignes de l'enseignant. 


\section{eJRIEPS 29 avril 2013}

\section{Conclusion}

Dans cette étude, en accord avec les présupposés de la théorie de l'enaction (Varela, 1989 ; Varela et al., 1993), nous avions comme perspective de ne pas réduire a priori les émotions à des causes ou des conséquences de l'apprentissage des élèves, mais de les considérer, lorsqu'elles étaient significatives pour eux, comme des expériences au cours desquelles des connaissances se transformaient. L'objectif de cette étude était essentiellement de décrire et spécifier ces phénomènes, à titre exploratoire, dans le cadre spécifique de leçons d'EPS visant l'acquisition par les élèves de techniques d'esquimautage en kayak. Les différentes séquences émotionnelles que nous avons analysées renforcent l'hypothèse selon laquelle les émotions ne font pas que faciliter ou inhiber les apprentissages. Cette étude accrédite l'idée selon laquelle les émotions, qu'elles soient ressenties comme agréables ou désagréables, jouent un rôle central dans la façon dont les élèves définissent leur propre situation. Elles peuvent amplifier les expériences et constituer, en elles-mêmes, des éléments de connaissance. Les spécificités de la pratique du kayak de mer et le nombre réduit de participants nous conduisent cependant à être prudents quant à la possibilité de généralisation de ces résultats, et quant à l'extension des perspectives pédagogiques avancées à d'autres situations scolaires. Dans cette étude, nous nous sommes attachés à analyser en adoptant un grain fin de description la transformation locale des connaissances au cours de séquences émotionnelles. Ces résultats, bien que limités, s'avèrent suffisamment heuristiques et féconds pour nous inciter à la mise en œuvre de recherches futures dans cette même perspective théorique et méthodologique, portant cependant sur des empans temporels plus larges, et concernant un nombre d'élèves plus important.

\section{Bibliographie}

Adler, P.A., \& Adler, P. (1987). Membership roles in field research. London : Sage Publications.

Barrett, L. F. (2008). The Science of Emotion : What People Believe, What the Evidence Shows, and Where to Go From Here. In Cognitive and Sensory Sciences and Education (BCSSE). Human Behavior in Military Contexts, Board on Behavioral. 


\section{eJRIEPS 29 avril 2013}

Washington: The National Academies Press, pp. 189-216. Bril, B. (2002). Apprentissage et contexte, Intellectica, 35, 251-268.

Berthoz, A. (2003). La décision. Paris : Odile Jacob.

Boekaerts, M. (2010). Motivation et émotion : deux piliers de l'apprentissage en classe. In Comment apprend-on ? : La recherche au service de la pratique. OCDE. http://dx.doi.org/10.1787/9789264086944-fr.

Bower, G.H., Monterot, K.P., \& Gilligan, S.G. (1978). Emotional Mood as a Context for Learning and Recall. Journal of Verbal Learning and Verbal Behavior, 17, 573585.

Bril, B. (2002). Apprentissage et contexte, Intellectica, 35, 251-268.

Bui-Xuan G., \& Haye, G. (2007). La sensibilité des enseignants d'EPS au plaisir des élèves. Enquête de I'AEEPS 2005-2007. In P. Gagnaire \& F. Lavie (Eds). Le plaisir des élèves en éducation physique et sportive. Futilité ou nécessité ? Montpellier : AEEPS/AFRAPS, 129-143.

Channouf, A. (2006). Les émotions - Une mémoire individuelle et collective. Bruxelles : Editions Mardaga.

Covington, M.V. (1992). Making the Grade : A Self-Worth Perspective on Motivation and School Reform. New York : Cambridge University Press.

Damasio, A. (2010). L'autre moi-même. Les nouvelles cartes du cerveau, de la conscience et des émotions. Paris : Odile Jacob.

Dan Glauser, E. (2009). Le sentiment subjectif. Intégration et représentation centrale consciente des composantes émotionnelles. In K.R. Scherer \& D. Sander (Eds.), Traité de psychologie des émotions (pp. 203-257). Paris : Dunod.

Delignières, D. (2003). Le plaisir en question. Revue Hyper, 222, 16.

Dougherty J.W.D. (Ed.) (1985). Directions in cognitive Anthropology. Chicago : University of Chicago Press.

Duncan, S., \& Barrett, L.F. (2009). Affect is a form of cognition : A neurobiological analysis. Cognition \& Emotion, 21, 1184-1211.

Durand, M. (2001). Chronomètre et survêtement. Paris : Éditions EP\&S.

Durand, M. (2008). Un programme de recherche technologique en formation des adultes. Une approche enactive de l'activité humaine et l'accompagnement de son apprentissage-développement, Education et Didactique, 2(2), 69-93. 


\section{eJRIEPS 29 avril 2013}

Edelman, G. M. (1987). Neural Darwinism : The theory of neuronal group selection. New York : Basic Books.

Fredrickson, B.L. (1998). What good are positive emotions ? Review of General Psychology, 2, 300-319.

Frijda, N.H. (1986). The Emotions. Cambridge : Cambridge University Press.

Gagnaire, P., \& Lavie, F. (2005). Cultiver les émotions des élèves en EPS. In L. Ria (Ed.), Les émotions (pp. 11-30). Paris : Éditions EP\&S.

Gagnaire, P., \& Lavie, F. (2010). Plaisir en EPS. Revue Hyper, 249, 13-23.

Grandjean, D., \& Scherer, K.R. (2009). Théorie de l'évaluation cognitive et dynamique des processus émotionnels. In D. Sander, \& K.R. Scherer (Ed.), Traité de psychologie des émotions (pp. 41-76). Paris : Dunod.

Horcik, Z., \& Durand, M. (2011). Une démarche d'ergonomie de la formation : Un projet pilote en formation par simulation d'infirmiers anesthésistes, @ctivités, 8(2), 173188.

Lafont, L., Cicero, C., Martin, L., Vedel, A., \& Viala, M. (2005). Apports de la psychologie sociale à l'intervention en EPS : rôle des interactions tutorielles et des "coping " modèles. eJRIEPS, 8, 89-102.

LeDoux, J. (2003). Neurobiologie de la personnalité. Paris : Odile Jacob.

Livet, P. (2002). Emotions et rationalité morale. Paris : PUF.

Marc, J, Grosjean, V., \& Marsella, M.C. (2011). Dynamique cognitive et risque psychosociaux : isolement et sentiment d'isolement au travail. Le Travail Humain, 74,(2), 107-130.

Masciotra, D., \& Morel, D. (2011). Apprendre par l'expérience active et située. La méthode ASCAR. Québec : Presse de l'Université du Québec.

Mogg, K., Millar, N., \& Bradley, B.P. (2002). Biases in eye movements to threatening facial expressions in generalized anxiety disorder and depressive disorder. Journal of Abnormal Psychology, 109, 695-704.

OCDE (2010a). Comment apprend-on ? : La recherche au service de la pratique. OCDE. http://dx.doi.org/10.1787/9789264086944-fr.

OCDE (2010b). Résultats du PISA 2009. OCDE.

Phelps, E. A. (2006). Emotion and cognition : Insights from studies of the human amygdala. Annual Review of Psychology, 57, 27-53. 


\section{eJRIEPS 29 avril 2013}

Ria, L. (2007). Les émotions au cœur de l'activité des enseignants débutants : description d'un observatoire de recherche en ergonomie cognitive. Les cahiers du CERFEE, 23, 101-121.

Ria, L., \& Récopé, B. (2005). Les émotions comme ressort de l'action. In L. Ria (Ed.), Les émotions (pp. 11-30). Paris : Éditions EP\&S.

Ria, L., Sève, C., Saury, J., Theureau, J., \& Durand, M. (2003). Beginning teacher's situated emotions : study about first classroom's experiences. Journal of Education for Teaching, 29, 219-233.

Ryan, R.M., \& Deci, E. (2000). Intrinsic and Extrinsic Motivations : Classic Definitions and New Directions. Contemporary Educational Psychology, 25, 54-67.

Rosch, E. (1978). Principles of categorization. In E. Rosch, B.B. Llyod (Eds.), Cognition and categorization (pp. 27-48). Hillsdale, NJ : Lauwrence Erlbaum Associates.

Sartre, J.P. (1995). Esquisse d'une théorie des émotions. Paris : Hermann.

Saury, J., \& Rossard, C. (2009). Les préoccupations des élèves durant des tâches d'apprentissage coopératives et compétitives en badminton : une étude de cas. Revue des Sciences de l'Education, 35(3), 195-216.

Saury, J., Adé, D., Gal-Petitfaux, N., Huet, B., Sève, C., \& Trohel, J. (2013). Actions, significations et apprentissages en EPS. Une approche centrée sur le cours d'expérience des élèves et des enseignants. Paris : Éditions EP\&S.

Sève, C. (2001). Emotion, action et intention dans l'activité de pongistes experts dans des matchs internationaux. Communication présentée au Séminaire Interdisciplinaire de Sciences et Technologies Cognitives : cognition, émotion et désir technique. Compiègne, France.

Sève, C., Ria, L, Poizat, G., Saury, J., \& Durand, M. (2007). Performance-induced emotions experienced during high-stakes table tennis matches. Psychology of Sport and Exercise, 8, 25-46.

Sève, C., \& Saury, J. (2010). Un programme de recherche en STAPS fondé sur la théorie du cours d'action. eJRIEPS, 20, 93-108.

Sève, C., Saury, J., Theureau, J., \& Durand, M. (2002a). Activity organization and knowledge construction during competitive interaction in table tennis. Cognitive Systems Research, 3, 501-522. 


\section{eJRIEPS 29 avril 2013}

Sève, C., Saury, J., Theureau, J., \& Durand, M. (2002b). La construction des connaissances chez les sportifs lors d'une interaction compétitive. Le Travail Humain, 65, 159-190.

Talarico, J.M., \& Rubin, D.C. (2003). Confidence, not consistency, characterizes flashbulb memories. Psychological Science, 14, 455-461.

Theureau, J. (1992). Le cours d'action : Analyse sémio-logique. Essai d'une anthropologie cognitive située. Berne : Peter Lang.

Theureau, J. (2004). Le cours d'action : Méthode élémentaire. Toulouse : Octarès.

Theureau, J. (2006). Le cours d'action : Méthode développée. Toulouse : Octarès.

Tran, V. (2009). Les émotions dans le monde de l'entreprise et du travail. In D. Sander, \& K.R. Scherer (Ed.), Traité de psychologie des emotions (pp. 333-358). Paris : Dunod.

Varela, J. (1988). Le cercle créatif. In P. Watzlawick (Ed.), L'invention de la réalité (pp. 329-345). Paris : Seuil.

Varela, F. (1989). Autonomie et connaissance. Essai sur le vivant. Paris : Seuil.

Varela, F.J., Thompson, E., \& Rosch, E. (1993). L'inscription corporelle de l'esprit. Sciences cognitives et expérience humaine. Paris : Seuil.

Weiner, B. (2007). Examining Emotional Diversity in the Classroom : An Attribution Theorist Considers the Moral Emotions. In P. Schutz, R. Pekrun, \& G. Phye (Ed.), Emotion in Education (pp. 75-88). San Diego : Academic Press, San Diego. 